\title{
Chairside options for the treatment of complete denture problems associated with the atrophic (flat) mandibular ridge
}

\author{
K. W. Tyson, ${ }^{1}$ and J. F. McCord, 2
}

This article outlines a number of simple clinical steps which should enable general dental practitioners to diagnose and treat the majority of complete denture problems in patients with atrophic mandibular ridges. The guiding principle is the reduction of forces transmitted to the denture-bearing area via the lower denture. Methods of optimising the size of the denture bases and/or the occlusal tables are discussed and illustrated.

\begin{abstract}
This paper is a sequel to a publication on 1 the management of patients with atrophic (flat) mandibular ridges. ${ }^{1}$ The first paper described an impression technique which permitted the clinician to determine, immediately after developing the working impression, how the denture-bearing tissues might tolerate firm pressure. The purpose of this paper is to suggest methods whereby modification of denture form may be made, often at the chairside, to improve patient tolerance of complete lower dentures.
\end{abstract}

\section{The clinical problem}

In the atrophic mandible, one of the principal functional problems, other than instability, arises from the inability of the residual ridge and its overlying tissues to withstand masticatory forces. ${ }^{1,2}$ Clinical experience indicates that the nature of the mucosa overlying the atrophic mandibular ridge influences a patient's ability to withstand loading. The loading of the mucosa overlying the mandibular bone is via the denture itself, and may occur during swallowing, mastication or clenching. ${ }^{1}$ Greater loads may occur if parafunction is present.

\footnotetext{
${ }^{1}$ Senior Lecturer in Prosthodontics, Department of Prosthetic Dentistry, University of Edinburgh, High School Yards, Edinburgh EH8 9XP ${ }^{2}$ Professor of Restorative Care of the Elderly, Unit of

Prosthodontics, University Dental Hospital of Manchester, Manchester M15 6FH REFEREED PAPER

Received 31.03.99; accepted 18.06.99

(C) British Dental Journal 2000; 188: 10-14
}

The mucosa is sandwiched between the denture base and the underlying bone so that all the forces generated by the mandible, during function and parafunction, are transmitted through this atrophic tissue.

The forces acting on the denture-bearing tissues have vertical, lateral and protrusive components, and it is necessary to consider parameters which may have an effect on the mucosa overlying the mandibular ridge. In essence, the following factors need to be considered: ${ }^{3}$

- Muscular control

- Vertical dimension of occlusion

- Area of the impression surface of the denture

- Size or area of the occlusal table

- Morphology of the occlusal table

- Occlusal balance.

In brief

- This article deals with how to deal with a common clinical problem the difficult complete lower denture.

- Suggestions are made regarding the diagnosis and management of certain situations and in so doing this article should help general dental practitioners to treat this common complaint with more confidence.

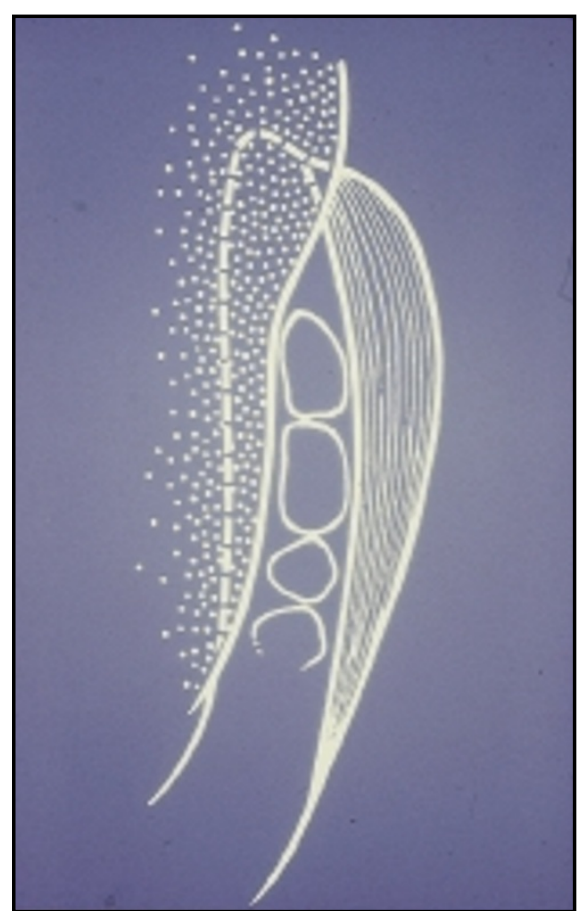

Fig. 1 Occlusal view of the desired relationship of the lower complete denture and the circumdenture musculature

\section{Muscular control}

Fish described the three surfaces of dentures as the impression (fitting) surface, the polished surface and the occlusal surface. ${ }^{4}$ In the interests of stability, and to reduce displacing forces, the polished surfaces should harmonise with the investing musculature, the tongue, lips and cheeks. ${ }^{5}$ This is shown in Fig. 1.

\section{Occlusal vertical dimension}

A common cause of excessive loading on the tissues is an excessive occlusal vertical dimension (OVD); this has been reported to be a major fault of dentures in a recent survey. ${ }^{6}$

Excessive OVD is often understood to mean that there is no freeway space (FWS). 
This can exacerbate a decreased capacity of the denture-bearing tissues to withstand loading. When the biological age of the patient's soft tissues exceeds the chronological age, there is often justification in increasing the FWS by $2-3 \mathrm{mms}$. In this way, although there is a reduction, physiologically, in the power of elevation of the mandible, there is a more realistic capacity of the tissues to tolerate masticatory loads.

\section{Area of the impression surface of the denture}

The pressure $(\mathrm{P})$ on an object is defined as the force or load (F) per unit area (A) and is represented by the equation $\mathrm{P}=\mathrm{F} / \mathrm{A}$. The pressure on the denture-bearing tissues is therefore affected by variations in either the load or the area. Any reduction in the area of the denture-base in contact with the mucosa, therefore, tends to increase the resultant pressure on the mucosa. As patient biting force, for the purposes of this discussion, may be considered to be essentially constant, the major factor influencing the pressure on the mucosa during function is the denture-base. In other words the smaller the fitting surface, the greater the mucosal loading - this was discussed in an earlier paper. $^{1}$

\section{Area of the occlusal table}

It is not uncommon to find relatively large, anatomically-shaped posterior teeth on complete dentures and although these teeth may appear 'natural', it is not reasonable to expect a patient with an atrophic mandibular ridge to function competently with such a large occlusal table. ${ }^{7}$ The greater the area of the occlusal table, the greater will be the effort to drive the denture teeth through a bolus of food. ${ }^{3}$

Another disadvantage of some anatomically-shaped posterior teeth is that they tend to be bulbous and so overhang the lingual flange. This situation is not conducive to good tongue control of the denture and causes complaints of looseness and instability.

\section{Morphology of the occlusal table}

Philosophies abound concerning the selection of posterior teeth for complete dentures, ranging from the choice of anatomical-shaped teeth with cusps to provide a balanced articulation to cuspless teeth to avoid occlusal locking. The choice of posterior teeth must take into consideration the masticatory characteristics of the patient. For example, an individual who makes purely vertical mandibular movements (a 'chopper'), does not require denture teeth with cusps to help shear a bolus with lateral movements; but if ruminatory mandibular movements occur in masticatory function (a 'grinder'), a balanced articulation is required to maintain denture stability. Selection of large, prominent cusps may induce denture instability and any induced lateral forces required to shear a bolus are transmitted through the mucosa.

\section{Occlusal balance}

Using only vertical movements, 'choppers' only need even contact or a balanced occlusion in the retruded contact position (RCP) and this is all that the clinician need prescribe. This should be simple to achieve by all technicians and should not be demanding of much laboratory time.

On the other hand 'grinders', with their mixture of vertical, lateral and protrusive movements should be provided with a balanced articulation (BA). Building a BA requires skill on the part of the technician and takes much longer; as a consequence, this will attract a higher laboratory fee.

\section{Dealing with the problems}

The problem facing the prosthodontist is whether to improve the presently unacceptable lower prosthesis and, following an assessment of the ability of the patient to con- trol a modified prosthesis, must decide on further modification or renewal. If the denture is reasonable in all aspects except for pain during mastication, then the problem is most probably one of support. In this case, modifying the denture may be the treatment of choice.

There are four areas to consider which involve the factors previously discussed:

\section{Facilitating muscular control by} improving the stability and control of the lower denture (by reduction of displacing forces)

In atrophic ridges, there is often a need to mould the peripheral form of the polished surfaces to be in harmony with the buccinator muscles. ${ }^{5}$ Figure $2 \mathrm{a}$ shows the recommended convex form of the periphery of a mandibular denture for a resorbed mandible. The importance of this basal convex form is two-fold:

(i) The cheeks can impart a downward component to the denture, assisting retention, as the buccal surface is now in an area where the buccinator can act upon it.

(ii) By filling in this space, it helps prevent 'dead space' where food debris may gather.

In theory, this is principally achieved at the time of recording the definitive impression, although the clinician may wish to alter the contour of the processed denture at the chairside. This procedure is described in factor 3, below.

This modification of the polished surfaces will present a more favourable profile of the

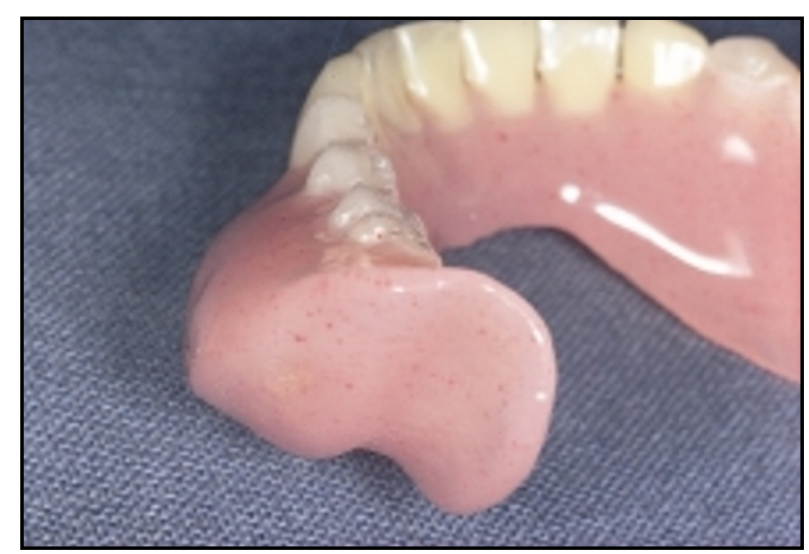

Fig. 2a Desirable form of posterior buccal flange of a complete lower denture which restores an atrophic mandibular ridge 
buccal aspect of the denture with regard to denture stability in relation to the buccinator muscles.

On the lingual aspect, any occlusal undercuts may be removed to enable the tongue to retain the denture rather than unseat it (Figs. 2b and 2c). This will also narrow the bucco-lingual diameter of the posterior teeth giving the added benefit of reducing the occlusal area.

\section{Correcting the occlusal vertical dimension}

Clinical studies have shown increased (excessive) OVD to be a common fault of many dentures. ${ }^{6}$ The measurement of OVD is established by recording the resting facial height (RFH) and subtracting from this the proposed freeway space (FWS). The measurements may be done with a Willis gauge, dividers or any vertical measuring tool which assesses the height of the lower third of the face. Guidelines suggest $2-5 \mathrm{~mm}$ for freeway space but this may need to be increased in older patients or for those patients with atrophic mucosa overlying the residual ridges. ${ }^{5}$

As RFH and thus VDO may be affected by the position of the head, the clinician is advised to record these values with the patient seated and looking straight ahead.

Excessive vertical dimension can only be reduced in the completed dentures by grinding the occlusal surfaces or by stripping off and resetting the posterior teeth.

Patients in the category of increased OVD generally report a history of the denture being comfortable on insertion but that an ache or burning sensation develops after several hours of wearing. Pain on eating is not a sine qua non (it is reasonable to surmise that the normal masticatory forces are less than the loads produced by the clenching resulting from the loss of freeway space). The lower denture usually becomes intolerable and patients commonly report that they have to remove their dentures by late afternoon. Such a complaint is usually pathognomonic of insufficient freeway space, in which case, occlusal modification or a reset is indicated.

If sufficient freeway space is present then, where there is pain on eating, consideration should be given to either increasing the denture bearing area (DBA) and/or reducing

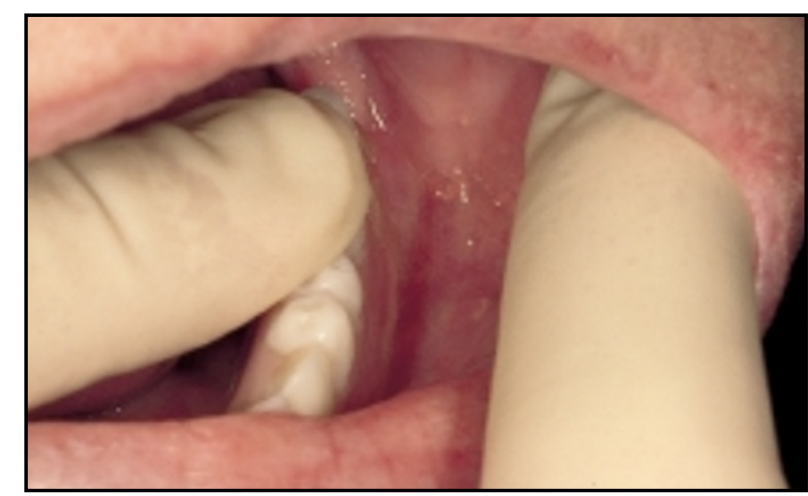

Fig. $2 b$ The occlusal table proved to be a problem because the wide posterior teeth presented lingual undercuts. In addition, the length of the table, extending over the ascending portion of the ridge, adversely affected denture stability

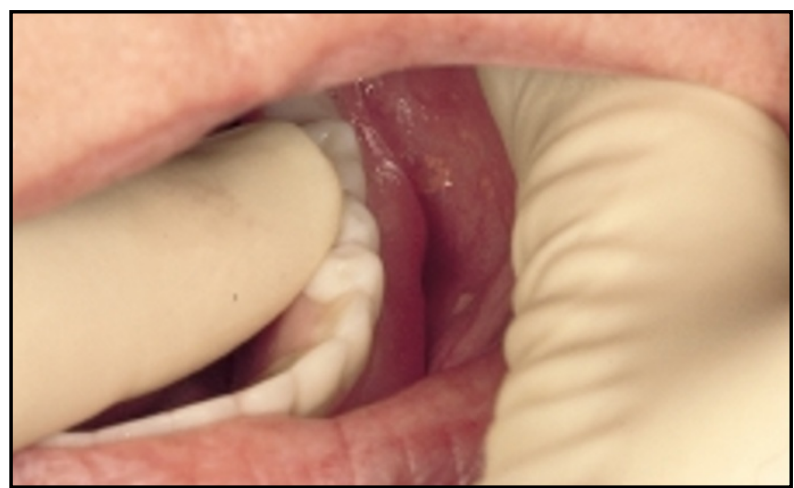

Fig. $2 c$ The occlusal table has been reduced significantly by reducing the length and width of the posterior table

the size of the occlusal table (see factor 3, below).

3. Reducing the forces required to drive the (lower) denture teeth through the bolus of food

This may be achieved by either increasing the DBA or reducing the size and morphology of the occlusal table.

(i) Increasing the DBA. Although prosthodontic norms recommend full use of the functional denture bearing area, this is rarely achieved. ${ }^{6}$ A consequence of this is that the smaller the size of the fitting surface of the denture, the greater are the loads applied to the underlying mucosa. In such cases, the denture bearing area may be increased using greenstick impression material before relining or by using a chairside lining material prior to the denture being relined conventionally (Fig. 3a). ${ }^{5}$ The former may be carried out using greenstick tracing compound (Kerr UK Ltd, Peterborough, UK) and subsequently recording a reline impression, while the latter may be by an appropriate chairside reline material such as Tokuso Rebase (Tokuyama Corp, Tokyo, Japan). Figure $3 \mathrm{~b}$ shows how the clinician may increase the denture-bearing area through the use of a chairside reline material.

(ii) Reducing the size and morphology of the occlusal table. Clinical experience indicates that many complete lower dentures have posterior teeth set without consideration of possible support problems. In general, occlusal tables tend to be too large. This leads to problems of support and stability which, singly and in combination, put too much pressure on the atrophic mucosa during function. ${ }^{8}$

A typical example of this is seen in Fig. 2b, where the lower second molar teeth are placed on what is obviously the ascending part of the ridge. This has been shown to have an adverse effect on denture stability. ${ }^{9}$ In addition, undercuts are present lingually which would aggravate the instability of the denture by causing the denture to tip up when the patient raises the tongue. Figure $2 \mathrm{c}$ shows how, in addition to decreasing the size of the occlusal table, such a modification will 


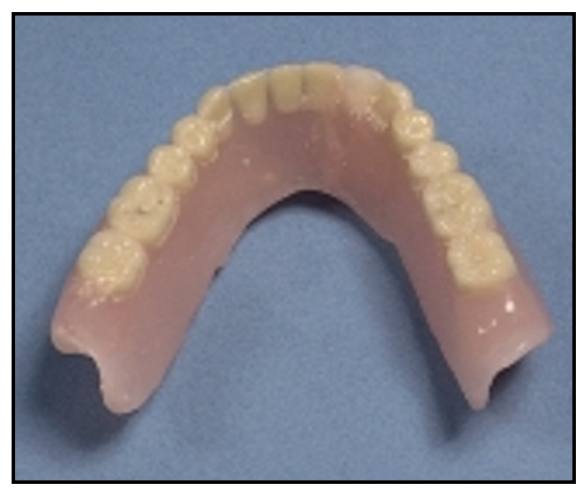

Fig. 3a Less than ideal form of a complete lower denture. The left flange is clearly not adequately using the maximum denture bearing area into and onto the buccal shelf

enhance stability by decreasing displacing forces.

Reduction of the occlusal table, by the removal of the second molar, is an effective way of reducing the occlusal table and there is no scientific evidence to support any advantage in the placement of two molar teeth and two premolar teeth in each posterior quadrant of a complete denture. The combination of reduced occlusal table and, if necessary, increased denture bearing area can greatly reduce the load per unit area (ie pressure) on the underlying mucosa and improve denture comfort, always assuming that the OVD is not excessive.

4. Eliminating disruptive occlusal contacts which lead to denture instability

Disruptive occlusal contacts may be present in any border position and in 'normal'



Fig. 3b Result of chairside 'reline' to improve the form of the buccal architecture of the lower denture and also to increase the denture-bearing area

function as well as parafunction. Their detection and elimination require clinical techniques which are outlined in most standard textbooks of prosthodontics. Where changes in OVD and elimination of such disruptive forces are indicated, occlusal pivots can be of great benefit. When they were originally described, ${ }^{10}$ temporary occlusal pivots were made on existing mandibular dentures as follows:

i Grind down or remove the posterior teeth from the denture and adjust the lower anterior teeth if there is locking

ii Prepare two rectangles of tin foil (about $10 \mathrm{~mm}$ broad and $15 \mathrm{~mm}$ long)

iii Practice recording the RCP, concurrently stabilising the mandibular denture with the (gloved) forefingers along the buccal flanges of the lower denture iv Add two columns of auto-polymerising resin to the $\overline{65556}$ area of the mandibular denture, (these points represent the point of balance of the denture) and cover the columns with the tin foil to control the resin (Fig. 4a)

$v$ Place petroleum jelly over the occlusal surfaces of the maxillary denture to ensure no resin from the mandibular denture adheres to the maxillary denture. Ask the patient to close gently in RCP to the desired OVD

vi When the resin is quite firm, place the denture in a bowl of hot water to complete the cure, remove the foil and trim the pivots to shape

vi Detect and remove any occlusal prematurities and polish the pivots.

A polished pivotal appliance is shown in Figure $4 \mathrm{~b}$. Pivots may look unusual but it is remarkable how well patients use them. Any further adjustments to the OVD or occlusal stability are simply made.

When the patient has had all adjustments made and has been wearing the denture comfortably for a period, it is a simple procedure to register the RCP with an acceptable registration material placed before and behind the pivots so that posterior teeth can be put in place. If new (replacement) dentures are to be made then the correct OVD has already been established.

Since pivots were first advocated, there have been changes in patient attitudes to care and medico-legal issues would appear to be assuming greater significance. For this reason, it may be wise to make a 'copy' of the patient's denture and
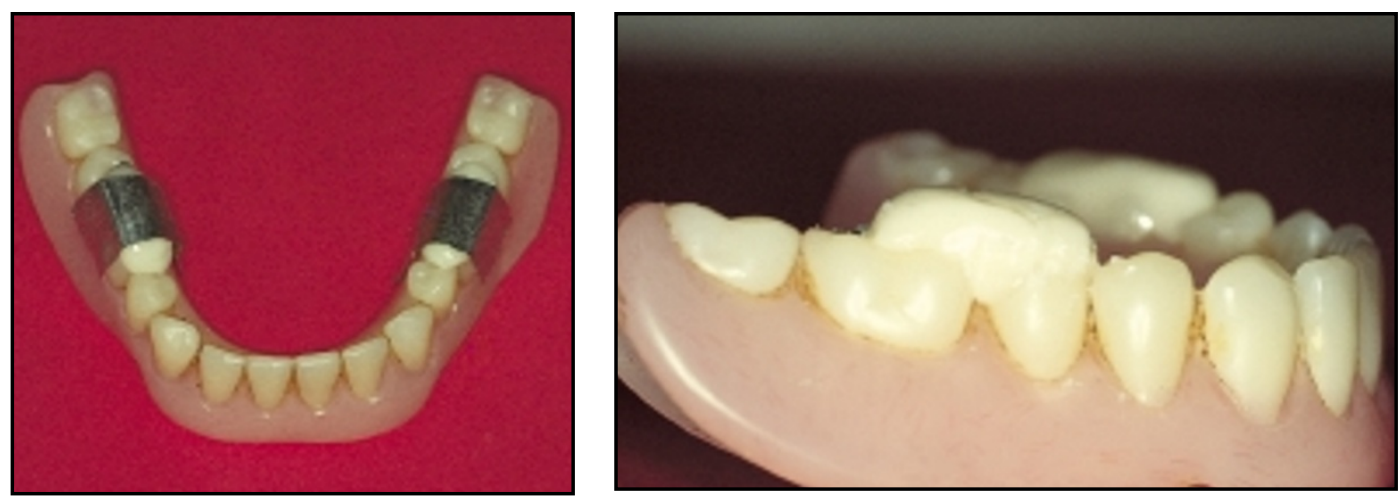

Fig. 4a (left) Tin foil placed over the two pillars of auto-polymerising resin in the pivotal areas of the lower denture; Fig. 4b (right) View of pivots prior to trimming and polishing 
make pivots on the copy, as the original denture can be returned intact to the patient if the clinician feels it to be necessary.

\section{Summary}

This article has shown relatively simple yet effective clinical techniques whereby a denture resting on an atrophic (flat/ depressed) mandibular ridge may be modified to improve function.

The creation of the correct form of the polished surfaces, a reduced occlusal table, an increased fitting surface, an appropriate vertical dimension and a free occlusion should result in comfortable lower dentures for many patients with atrophic mandibular ridges.

1 McCord J F, Tyson K W. A conservative prosthodontic option for the treatment of edentulous patients with atrophic (flat) mandibular ridges. Br Dent J 1997; 182: 469-472.

2 Zarb G A, Bolender C L, Hickey J C, Carlsson G C. Boucher's Prosthodontic Treatment for Edentulous Patients. 10th edn. pp 1-27. St. Louis: C.V.Mosby, 1990.

3 Watt D M, MacGregor A R. Designing Complete Dentures. 2nd edn. pp 43, 86. Bristol: Wright, 1986.

4 Fish F. Principles of Full Denture Prosthesis. 6th edn. pp32-66. London: Staples Press, 1964.
5 Grant A A, Heath J R, McCord J F. Complete Prosthodontics: Problems, Diagnosis and Management. pp 42,135. London: Wolfe, 1994.

6 Basker R M, Ogden A R and Ralph J P. Complete denture prescription - an audit of performance. Br Dent J 1993; 174: 278-284.

7 Lang B. Complete denture occlusion. Dent Clin N Am 1966; 40: 85-101.

8 Jacobson T E, Krol A J. A contemporary review of the factors involved in complete denture retention, stability and support. J Prosthet Dent 1983; 49: 5-15; 165-172; 306-313.

9 Jooste C H, Thomas C J. Complete mandibular denture stability when posterior teeth are placed over a basal tissue incline. J Oral Rehab 1992; 19: 441-448.

10 Watt D M, MacGregor A R. Designing complete dentures. pp 361-367. Bristol: W B Saunders, 1976

\section{BDA Information Centre Services}

\section{Did you know?}

- As a BDA member you can gain access to one of the best dental information services in the world

- You don't have to be based in London to use the service

- You can borrow books, videos and information packages

- You can borrow up to eight items via the postal system The only cost to you is the cost of the return postage. If you're not sure what to request then telephone us and we can advise you.

- You are entitled to free MEDLINE searches Telephone us with a subject and we will send you a list of relevant references with abstracts.

\section{- You can request photocopies of journal articles}

There is a small charge for this service and you need to fill in a Photocopy Request Form first. Telephone us if you would like one of these forms.

- You can register to receive free Current Dental Titles These are MEDLINE-based lists of references on eight areas of dentistry which are sent to you automatically twice a year. Phone us for a registration form.

For further details of any of these services dial $01719350875 \times 265$. or contact us via e-mail at: Infocentre@bda-dentistry.org.uk Visit the Information Centre web pages at: www.bda-dentistry.org.uk 